\title{
The link between the genetic polymorphisms of the innate immune signaling molecular factors with periodontitis
}

\author{
Gurumoorthy Kaarthikeyan, ${ }^{1}$ Anandan Balakrishnan, ${ }^{2}$ Nadathur Doraisamy Jayakumar ${ }^{1}$ \\ ${ }^{1}$ Department of Periodontics, Saveetha Dental College, Saveetha University, Saveetha Institute of Medical and Technical \\ Sciences; ${ }^{2}$ Department of Genetics, AL Mudaliyar Post Graduate Institute of Basic Medical Sciences, Chennai, India
}

\begin{abstract}
Periodontitis is a chronic inflammatory disease causing destruction of supporting tissues of teeth. Even though the gramnegative anaerobes are essential for the initiation of periodontal destruction, multiple risk factors are essential for the progression of the disease. The genetic risk factor plays a significant role in the etiopathogenesis of periodontal disease. The innate immune mechanism is the first line of defense in screening and combating the invading periodontal pathogens. The genetic polymorphisms in the 3'UTR region of the innate immune signaling molecular factors like toll-like receptors, nod-like receptors and the polymorphisms in the epigenetic regulators of these factors like microRNA146a, apolipoproteinE might play an important role in the etiopathogenesis of periodontal destruction.
\end{abstract}

\section{Introduction}

Periodontitis is a chronic inflammatory disease of multifactorial etiology. In periodontitis there is loss of the supporting tis-

\footnotetext{
Correspondence: Gurumoorthy Kaarthikeyan, Department of Periodontics, Saveetha Dental College, Saveetha University, Saveetha Institute of Medical and Technical Sciences, 162 P.H. Road, Velappanchavadi, Chennai 6000077, India.

Tel.: +91.9841222027.

E-mail: drkarthik79@yahoo.co.in

Key words: Polymorphisms; microRNA; apolipoproteinE.

Contributions: the authors contributed equally.

Conflict of interest: the authors declare no potential conflict of interest.

Funding: none.
}

Received for publication: 21 November 2017.

Revision received: 11 June 2018.

Accepted for publication: 13 June 2018.

(C) Copyright G. Kaarthikeyan et al., 2018

Licensee PAGEPress, Italy

Journal of Biological Research 2018; $91: 7202$

doi:10.4081/jbr.2018.7202

This article is distributed under the terms of the Creative Commons Attribution Noncommercial License (by-nc 4.0) which permits any noncommercial use, distribution, and reproduction in any medium, provided the original author(s) and source are credited. sues of the teeth. Although the gram-negative anaerobes are essential for the initiation of the periodontal destruction, the course and propagation of the periodontal destruction is determined by multiple risk factors. ${ }^{1}$ There are various risk factors for periodontal disease like smoking, diabetes mellitus, genetics and stress. Among these risk factors, the genetic factor plays a major role in determining the host susceptibility to periodontal disease. $^{2}$

The most common periodontal pathogens include Porphyromonas gingivalis, Aggregatibacter actinomycetemcomitans, Tannerella forsythia, Treponema denticola. The main virulence factors of gram-negative periodontal pathogens like $P$. gingivalis, A. actinomycetemcomitans are lipopolysacharides (LPS). These pathogen associated molecular patterns (PAMPs) like LPS are screened by certain conserved pattern recognition receptors (PRR) on various cells. The main PRRs are toll-like receptors (TLRs). ${ }^{3}$ Among the various toll-like receptors, TLR4 plays a major role in detecting the gram negative anaerobes. Generally the TLR4 present on the cell surface screens for the LPS and it sends signals intracellularly either through myeloid differentiation factor (MyD88) dependent or MyD88 independent pathway. The MyD88 dependent pathway transmits the signal through Tumour necrosis factor Receptor Associated Factor6 (TRAF6), interleukin 1 receptor associated kinases (IRAK1) and finally leading to the stimulation of nuclear factor kappa B (NF$\mathrm{k} \beta$ ). The transcription factor $(\mathrm{NF}-\mathrm{k} \beta)$ enters the nucleus and activates the transcription of many proinflammatory cytokine genes. ${ }^{4}$ Similar to the toll-like receptors, the intracellular danger signals like danger associated molecular patterns (DAMPs) and intracellular pathogen associated components are screened by the inflammasomes called nod-like receptors (NLRs). ${ }^{5}$ The inflammasomes are multiprotein complexes that activates caspase 1 and in turn helps in the maturation of the pro interleukin 1 $\beta$ (IL-1 $\beta$ ). Four inflammasomes have been identified so far: NLRP1, NLRP3, NLRC4 and AIM2. Among these NLRs, NLRP3 plays a significant role in inflammation. ${ }^{6}$

This innate immune signaling mechanism ultimately leads to the secretion of proinflammatory cytokines, which in turn regulates the immune response to periodontal destruction. These signaling mechanisms are finely regulated by small non coding nucleotides known as micro RNAs (miRNA). ${ }^{7}$ The microRNAs are 20-23 nucleotides in length which post transcriptionaly regulates the genes. There are more than 2500 microRNAs have been identified so far. Among the various microRNAs, the microRNA146a has a significant role in the innate immune mechanism and it negatively regulates the TLR signaling pathway. ${ }^{8}$ This miR146a are in turn regulated by apoliprotein $\mathrm{E}$ (apoE) as shown in Figure 1. 


\section{Toll-like receptors}

The human TLRs comprises of ten related proteins. The gene for TLR4 is located on the long arm of the chromosome 9 (9q33.1). The gene of TLR4 has 13317 nucleotide bases and it consists of four exons. ${ }^{9}$ The TLR4 screens for the gram-negative anaerobes like Porphyromonas gingivalis, Tanerella forsythia, etc. In the case of microRNA146a, multiple components of proinflammatory signaling pathways (including TRAF6, IRAK1, IRAK2, MyD88, RelB.STAT1, CARD 10 and TLR4) have been identified as targets. ${ }^{10}$

\section{Nod-like receptors}

The main members of the nucleotide binding domain like receptor gene family includes the pyrin domain containing 1 family (NLRP1), pyrin domain containing 3 family (NLRP3), CARD domain containing 4 family (NLRC4). NLRP3 is a key molecule in the regulation of innate immune system. ${ }^{6}$ It plays a vital role in the recognition of microbial products and intracellular danger components. Once the NLRP3 gets stimulated, it leads to the assembly of an adaptor protein, apoptosis associated speck like protein containing a carboxyl terminal caspase recruitment domain and the effector protein caspase- 1 . This cleaves the pro interleukin 1 beta and interleukin 18 into their active mature forms. ${ }^{5}$ The NLRP3 gene which is also known as CIAS1 provides instruction for the synthesis of a protein called cryopyrin. This gene is located on the long arm of chromosome 1 (1q44).

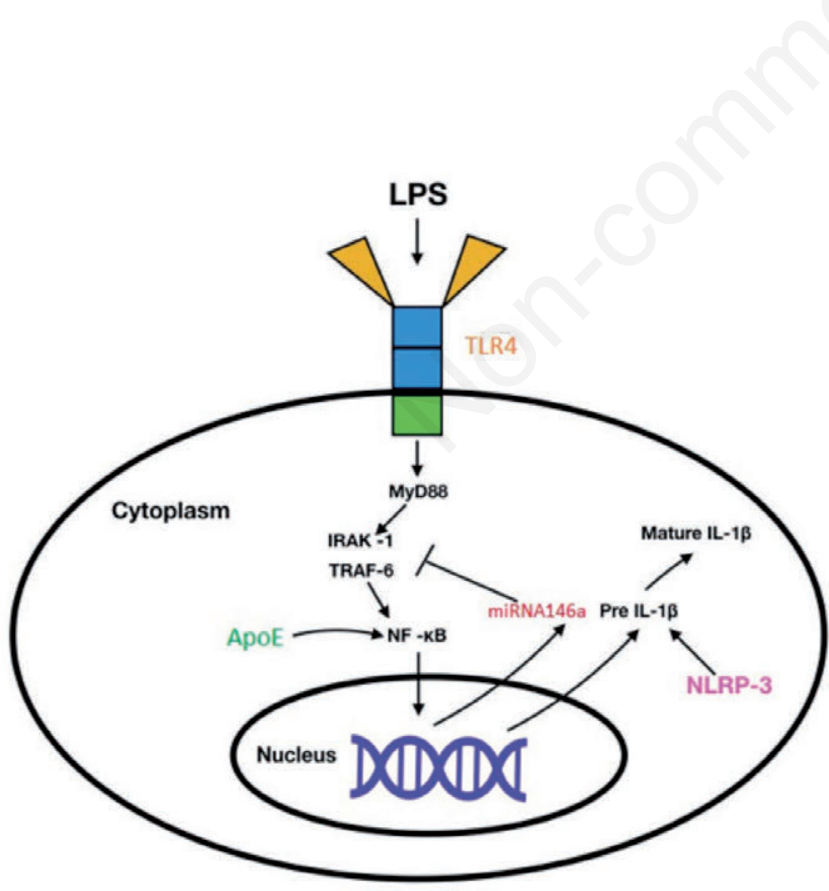

Figure 1. Toll-like receptors4 signaling pathway and its regulation by miRNA146a, apoliprotein E (ApoE) and Nod-like receptors pyrin 3 (NLRP3). LPS, lipopolysaccharides; IRAK1, interleukin 1 receptor associated kinases; TRAF-6, tumour necrosis factor Receptor Associated Factor6; MyD88, myeloid differentiation factor.

\section{Micro RNA146a}

The gene coding miRNA 146a is located on the chromosome $5 q 33.3$ [Loc 285628]. The gene consists of two exons separated by approx $16 \mathrm{~kb}$ of genomic sequence with the mature miRNA146a situated in the second exon. miRNA146a promoter is found in the upstream 550bp and its LPS responsiveness is totally dependent on the NF-k $\beta$ binding sites. The mature miRNA146a has the following nucleotide sequence 5'-UGAGAACUGAAUUCCAUGGGUU-3' (mature miRNA146a).

miR146a plays a major role in the regulation of TLR4 signalling pathway. It negatively regulates the inflammatory pathway by feedback loop inhibition. The miR146a blocks the IRAK1, TRAF6 and also binds to the 3'UTR of TLR4. ${ }^{10}$

\section{Apolipoprotein E}

Apolipoprotein E (ApoE) gene is located on the long arm of the chromosome 19(19q13.2). This gene consists of four exons and three introns. ApoE gene is polymorphic at two single nucleotides (rs429358 and rs7412), resulting in three different alleles $(\varepsilon 2, \varepsilon 3$ and $\varepsilon 4)$ and six $A P O E$ genotypes $(\varepsilon 2 / \varepsilon 2, \varepsilon 2 / \varepsilon 3, \varepsilon 2 / \varepsilon 4, \varepsilon 3 / \varepsilon 3, \varepsilon 3 / \varepsilon 4$ and $\varepsilon 4 / \varepsilon 4) .{ }^{11}$ The frequency of occurrence of the APOE alleles varies among different ethnic populations and the general frequency of $\varepsilon 2, \varepsilon 3$ and $\varepsilon 4$ allele is $8.4 \%, 77.9 \%$ and $13.7 \%$, respectively. ${ }^{12}$ Human apoE is a $34.2 \mathrm{kDa}$ glycoprotein with 299 amino acid residues. The aminoacid residues at 112 and 158 are either cysteine or arginine which determines the three isoforms of apoE. The single amino acid differences among the three apoE isoforms alter the protein's structure and influence its lipid association and receptor binding. ${ }^{13}$ ApoE is a major cholesterol carrier and plays an important role in maintaining lipid homeostasis. ApoE is produced by liver, brain, spleen, kidney, lung and muscle tissues. Hepatic parenchyma cells produce $2 / 3$ to $3 / 4$ of the apoE in plasma. ${ }^{11}$ ApoE2 transports lipids less efficiently, and $\varepsilon 2$ homozygosity is associated with an increased risk for type III hyperlipoproteinemia. ApoE selectively regulates TLR4- and TLR3-mediated signaling of IL-12 production and apoE may suppress the Th1 immune response by modulating IL- 12 production. ${ }^{14}$ The physiological properties of apoE, such as antioxidant, antiapoptotic, immunomodulatory and atheroprotective capacities are significantly influenced by apoE polymorphism. ${ }^{14-16}$

\section{Hypotheses}

The receptors involved in the innate immune mechanisms like TLRs,NLRPs and the epigenetic regulators like micro RNAs, apoE might determine the course and progression of inflammatory pathway. Thus a new hypothesis can be framed that the genetic polymorphisms in the 3'UTR of the major innate immune receptors like TLR4, NLRP3 and the SNPs within the miR146a, apoE genes might be associated with the inflammatory disease like periodontitis.

There are contradictory results regarding the association of TLR4 gene polymorphisms with periodontitis in different ethnic populations. ${ }^{17}$ But there are no studies correlating the link between 3'UTR gene polymorphisms of TLR4 with periodontitis. Because, the miRNAs generally binds to the 3'UTR of various mRNAs and regulate them by translational repression, mRNA cleavage or 
mRNA decay depending on the degree of complementarity of the specific target mRNA. The miR146a regulates the innate immune mechanism by targeting the signaling factors like TRAF6, IRAK1 and the PRRs like TLR4 gene. The partial complementarity required for the successful binding of miR146a with mRNAs of TRAF6, IRAK1, TLR4 might be altered when there is single nucleotide polymorphisms in the miR146a and 3'UTR of TLR4 gene. Some studies have found an association of miRNA146a gene polymorphism with periodontitis in southIndian and Iranian population. ${ }^{18,19}$ This microRNA146a is in turn regulated by the apolipoprotein E. ApoE regulates inflammation by suppressing the $\mathrm{NF}-\mathrm{k} \beta$ signaling. ApoE increases the expression of the transcription factor PU.1 and which in turn increases miRNA146a levels to suppress the NF-k $\beta$ signaling. Thus the genetic polymorphisms of ApoE might influence the inflammatory status of the periodontium. ${ }^{14,20,21}$

\section{Conclusions}

Thus the genetic changes/polymorphisms of the components of innate immune mechanisms like TLR4, NLRP3 and the epigenetic regulators of this pathway like miR146a and ApoE might play a vital role in the etiopathogenesis of the inflammatory disease Periodontitis. Further studies are required to confirm this hypothesis in different ethnic groups.

\section{References}

1. Darveau RP. Periodontitis - a polymicrobial disruption of host homeostasis. Nat Rev Microbiol 2010:8:481-90.

2. Kinane DF, Shiba H, Hart TC. The genetic basis of periodontitis. Periodontol 2000 2005;39:91-117.

3. Takeda K, Akira S. Toll-like receptors in innate immunity. Int Immunol 2005; 17:1-14.

4. Kawai T, Akira SO. The role of pattern recognition receptors in innate immunity - an update on toll-like receptors. Nat Immunol 2011;11:373-84.

5. Martinon F, Mayor A, Tschopp J. The inflammasomes: guardians of the body. Ann Rev Immunol 2009;27:229-65.

6. Pedra JH, Cassel SL, Sutterwala FS. Sensing pathogens and danger signals by the inflammasome. Curr Opin Immunol 2009;21:10-6.

7. Quinn SR, O' Neill LA. Atrio of microRNAs that control Toll- like receptors signaling. Int Immunol 2011;23:421-5.

8. Chatzkyriakidou A, Voulgari PV, Georgiou I, Drosos AA. The role ofmiR146a and its target IL-1Rassociated kinase (IRAK1) in psoriatic arthritis susceptibility. Scand J Immunol 2010;71:382.

9. O'Neill LAJ, Golenbock D, Bowie AG. The history of toll-like receptors - redefining the innate immunity. Nat Rev Immunol 2013;13:453-60.

10. Cheng HS, Njock MS, Khyzha N, et al. Non coding RNAs regulate NF-Kb signaling to modulate blood vessel inflammation. Front Genet 2014;5:422.

11. Mahley RW. Apolipoprotein E: cholesterol transport protein with expanding role in cell biology. Science 1988;240:622-30.

12. Corbo RM, Scacchp R. Apolipoprotein E (APOE) allele distribution in the world. Is APOE*4 a 'thrifty' allele? Ann Human Genet 1999;63:301-10.

13. Gerdes LU. The common polymorphism of apolipoprotein E: geographical aspects and new pathophysiological relations. Clin Chem Lab Med 2003;41:628-31.

14. Zhong H, Cai Y, Cheng J, et al. Apolipoprotein E epsilon 4 enhances the association between the rs 2910164 polymorphism of miR-146a and risk of atherosclerotic cerebral infarction. J Ateroscler Thromb 2016;23:819-29.

15. Anoop S, Misra A, Meena K, Luthra K. Apolipoprotein E polymorphisms in cerebrovascular and coronary heart diseases. Indian Med Res 2010;132:363-78.

16. Johnson LA, Arbones-Mainar JM, Fox RG, et al. Apolipoprotein E4 exaggerates diabetic dyslipidemia and atherosclerosis in mice lacking the LDL receptor. Diabetes 2011;60:2285-94.

17 Jin SH, Guan XY, Liang WH, et al. TLR4 polymorphisms and periodontitis susceptibility.A meta-analysis. Medicine 2016;95:4845-52.

18. Venugopal P, Lavu V, Ranga Rao S, Venkatesan V. Evaluation of a panel of single-nucleotide polymorphisms in mir-146a and mir-196a2 genomic regions in patients with chronic periodontitis. Genetic Test Mol Biomarkers 2017;21:228-35.

19. Kadkhodazadeh M, Jafari AR, Amid R, et al. MiR146a and MiR499 gene polymorphisms in iranian periodontitis and periimplantitis patients. J Long Term Effects Med Implants 2013;23:9-16.

20. Li K, Ching D, Raffai RL. Apolipoprotein E enhances microRNA-146a in monocytes and macrophages to suppress nuclear factor kappa beta driven inflammation and atherosclerosis. Circul Res 2015;117:e1-11.

21. Fish JE, Cybulsky MI. ApoE attenuates atherosclerosis via miR146a. Circul Res 2015;117:3-6. 